PHYSICAL REVIEW D 76104040 (2007)

\title{
Helical Symmetry in Linear Systems
}

\author{
Jiří Bičák $k^{1,2}$ and Bernd G. Schmidt ${ }^{2}$ \\ ${ }^{1}$ Institute of Theoretical Physics, Faculty of Mathematics and Physics \\ Charles University, Prague, Czech Republic \\ ${ }^{2}$ Max-Planck-Institut für Gravitationsphysik, Albert-Einstein-Institut \\ Am Mühlenberg 1, D-14476 Golm, Germany
}

\begin{abstract}
We investigate properties of solutions of the scalar wave equation and Maxwell's equations on Minkowski space with helical symmetry. Existence of local and global solutions with this symmetry is demonstrated with and without sources. The asymptotic properties of the solutions are analyzed. We show that the Newman-Penrose retarded and advanced scalars exhibit specific symmetries and generalized peeling properties.

PACS numbers: $04.20 .-\mathrm{q}, 03.50 .-\mathrm{z}$
\end{abstract}

\section{INTRODUCTION}

A beautiful theorem by Leon Lichtenstein demonstrates the existence of solutions describing the circular motion of two fluid bodies around their center of mass under the Newtonian force of gravity [1]. This solution is invariant under the symmetry generated by the vector field $\partial_{t}+\Omega \partial_{\phi}$, the helical symmetry.

Are there corresponding solutions in Einstein's theory of gravity? For truly isolated systems such solutions will not exist in General Relativity because the orbiting bodies will generate gravitational radiation and spiral towards each other. It is however conceivable - and this was first conjectured by Detweiler [2] — that if there is the right amount of incoming radiation which would just compensate the outgoing radiation, then the bodies could stay on a circular orbit and the spacetime would admit helical symmetry as an isometry.

There is an example of a solution with helical symmetry in Maxwell's theory by Schild [3]: He shows that two opposite electric point charges can move on a circular orbit if the force on one particle is given by the Lorentz force of the "one half advanced plus retarded field" of the other particle. In this solution the total energy of the field is infinite. (See also [4] where this is generalized to $N$ scalar point particles.)

One also expects that in a fully relativistic treatment of the 2-body problem with helical symmetry the ADM mass of the solution will be infinite and that the solutions will not be asymptotically flat in the usual sense at spatial or null infinity. Because of this it is not even clear how one defines "helical symmetry" if there is no asymptotic symmetry group to characterize the infinitesimal helical Killing field $\partial_{t}+\Omega \partial_{\phi}$.

Apart from this fascinating question whether Einstein's theory has really solutions with helical symmetry in which incoming radiation balances outgoing radiation, there is interest in the numerical community to use data with approximate helical symmetry in the numerical construction of solutions of the 2-body problem and the calculation of the radiation emitted. This question has lead to various both analytical and numerical studies of helical solutions of linear and nonlinear model problems by J. Friedman, R. Price, E. Gourgoulhon, their coworkers, and others (see, e.g., [5] - [9], and references therein). The primary goal here is computing quasiequilibrium configurations of close binary compact objects, or individual rotating neutron stars.

To address the problem of helical solutions in Einstein's theory it is absolutely necessary to have a really good understanding of the linear situation. The reason is that solutions to nonlinear problems are usually solved as a limit of a sequence of linear problems. No systematic study of linear helical solutions of the scalar wave equation, Maxwell's equations and linearized gravity is available. This is the topic of the present paper.

Section II studies solutions with helical symmetry i.e. solutions invariant under the symmetry generated by $\partial_{t}+\Omega \partial_{\phi}-$ of the scalar wave equation on Minkowski space. There is a very important point: because of the assumed symmetry one can study the wave equation on the space of orbits of the symmetry. There one obtains an apparently simpler 3-dimensional problem. However, the symmetry reduced wave equation is a second order PDE which changes its character: it is elliptic near the center and hyperbolic far out. No general theory is available in this situation. There is the paper by Torre [10] who obtains some existence results for the inhomogeneous equations on part of Minkowski space.

We proceed completely differently. A lot is known about the wave equation in Minkowski space. In particular, the retarded and advanced solutions of a spatially compact source with helical symmetry are also helical. So, with almost no work we obtain solutions of the inhomogeneous wave equation on the space of orbits. 
The general homogeneous solution with helical symmetry can be constructed via a spherical harmonics decomposition. So, we know the general solution.

There are helical solutions with explicit $Y_{l m}$ angular behaviour. We give an integral representation of such solutions.

In Section III we investigate the asymptotic behaviour of these solutions near spatial and null infinity. The retarded solution of a spatially compact helical source has the usual Bondi-type expansion [11] at future null infinity but no such expansion exists at past null infinity. The standard radiation field of the solution, as defined, e.g., in [11], 12], 13], does not exist on past null infinity. Hence for the retarded plus advance solution no radiation field is defined at all. Also, the decay at spatial infinity is rather weak, the field decays like $\sin r / r$.

In Section IV we calculate the retarded and advanced field of a scalar source moving on a circle with constant velocity. The rather complicated periodicity properties of the solutions become apparent.

The analogous solution in Maxwell's theory is presented in Section V. We give the fields in terms of the null tetrad NP (Newman-Penrose) projections, i.e., as three complex scalars. Since the charged particles moving in circular obits occur in synchrotrons (where they are guided by external magnetic fields) our final expressions might be of some use also elswhere. In Schild's work [3] the field is not given. The usual radiation field again does not exist but the NP scalars exhibit generalized peeling properties. As a consequence of the helical symmetry, the retarded and advanced NP scalars show specific symmetry properties.

From the results in Maxwell's theory one can almost guess the formulae for Einstein's linearized gravity if needed.

\section{THE WAVE EQUATION ON MINKOWSKI SPACE}

We begin by considering the wave equation on Minkowski space and want to describe global and local solutions with helical symmetry.

A function $F(t, r, \theta, \phi)$ (in standard spherical coordinates on Minkowski space) is invariant under $\partial_{t}+\Omega \partial_{\phi}$ if and only if

$$
F(t, r, \theta, \phi)=G(\phi-\Omega t, r, \theta)=G(\hat{\phi}, r, \theta) .
$$

The function $G$ is periodic in its first argument with pe$\operatorname{riod} 2 \pi . F$ is a solution of the scalar wave equation if and only if $G$ satisfies

$$
\begin{aligned}
-\Omega^{2} G_{, \hat{\phi} \hat{\phi}} & +G_{, r r}+\frac{2}{r} G_{, r}+ \\
& +\frac{1}{r^{2} \sin \theta}\left(\sin \theta G_{, \theta}\right)_{, \theta}+\frac{1}{r^{2} \sin ^{2} \theta} G_{, \hat{\phi} \hat{\phi}}=0 .
\end{aligned}
$$

(In Secs. II and III we set the speed of light $c=$ 1.) A remarkable property of this equation is that it changes its character! The norm of the helical Killing vector is $N=-1+\Omega^{2} r^{2} \sin ^{2} \theta$ and therefore the helical Killing vector is timelike near the axis $(N<0)$, null for $N=-1+\Omega^{2} r^{2} \sin ^{2} \theta=0$, and spacelike for $N=-1+\Omega^{2} r^{2} \sin ^{2} \theta>0$. This implies that the reduced equation (2) is elliptic near the center and hyperbolic for $N=-1+\Omega^{2} r^{2} \sin ^{2} \theta>0$. This raises interesting questions concerning solutions defined globally. We will denote the three regions as: $T=\{(\hat{\phi}, r, \theta) \mid N<0\}$, $L=\{(\hat{\phi}, r, \theta) \mid N=0\}, S=\{(\hat{\phi}, r, \theta) \mid N>0\}$.

\section{A. Local source-free solutions}

Obviously in $T$ local solutions can be determined by boundary value problems and in $S$ by Cauchy problems. What happens near $L$ is not so clear.

Suppose we have a $C^{2}$ solution of (2) defined for $r_{1}<$ $r<r_{2}$ such that $N$ changes its sign in this region. We can expand into spherical harmonics

$$
G(\hat{\phi}, r, \theta)=G(r) Y_{l m}(\hat{\phi}, \theta), \quad \hat{\phi} \in[0,2 \pi),
$$

and obtain for $G(r)$ the radial equation

$$
m^{2} \Omega^{2} G+\frac{1}{r}(r G)_{, r r}-\frac{1}{r^{2}} l(l+1) G=0 .
$$

The general solution in terms of spherical Bessel functions is

$$
G_{l m}(r)=\frac{a_{l m}}{\sqrt{m \Omega r}} J_{l+\frac{1}{2}}(m \Omega r)+\frac{b_{l m}}{\sqrt{m \Omega r}} Y_{l+\frac{1}{2}}(m \Omega r) .
$$

The set $L$, the light cylinder, is no singularity for the radial equation. Hence, we see that there are local solutions for which nothing particular happens at $L$.

The only solutions regular at the origin are given by $b=0$. For $m \Omega r \gg l$ the solutions behave as $\sin (m \Omega r-$ $\left.\frac{\pi}{2} l\right) / m \Omega r$.

Thus we know the general $C^{2}$ solutions in terms of converging superpositions of spherical harmonics and Bessel functions.

$$
G(\hat{\phi}, r, \theta)=\sum_{l, m} a_{l m} G_{l m}(r) Y_{l m}(\hat{\phi}, \theta) .
$$

The coefficients $a_{l m}, b_{l m}$ in (5) may depend on $\Omega$. Taking (5) in the form

$$
\begin{aligned}
G_{l m}(r)= & \frac{\bar{a}_{l m}}{(m \Omega)^{l}} \frac{1}{\sqrt{m \Omega r}} J_{l+\frac{1}{2}}(m \Omega r)+ \\
+ & \bar{b}_{l m}(m \Omega)^{l+1} \frac{1}{\sqrt{m \Omega r}} Y_{l+\frac{1}{2}}(m \Omega r),
\end{aligned}
$$


where $\bar{a}_{l m}, \bar{b}_{l m}$ are independent of $\Omega$, we find, using the properties of the Bessel functions, that in the limit $\Omega \rightarrow 0$

$$
G_{l m}(r)=\frac{\bar{a}_{l m}}{(2 l+1) ! !} r^{l}-\bar{b}_{l m}(2 l-1) ! ! \frac{1}{r^{l+1}} .
$$

This is the general static $Y_{l m}$-solution of the Laplace equation.

\section{B. Global source-free solutions}

Now we assume that we have a $C^{2}$ solution defined on Minkowski space. Again we can decompose into spherical harmonics and because of the regularity at the origin only $J$-Bessel functions appear:

$$
G(\hat{\phi}, r, \theta)=\sum_{l, m} a_{l m} \frac{1}{\sqrt{m \Omega r}} J_{l+\frac{1}{2}}(m \Omega r) Y_{l m}(\hat{\phi}, \theta) .
$$

This shows that a global $C^{2}$ solution is uniquely determined by its values in a neighborhood of the center.

What is the asymptotics of such solutions? From the $Y_{l m}$ decomposition it is not obvious what type of decay one can produce by superpositions. Remember that a superposition of not decaying plane wave solutions of the wave equation can produce solutions with spatially compact support.

There is an easy argument that we cannot obtain solutions of finite energy: suppose we have a $C^{2}$ solution of finite energy; then the energy in a finite box around some fixed point in space has to decay in time, a general property of solutions of the wave equation. Such a decay contradicts helical symmetry.

In particular, there are no $C^{2}$ solutions with spatially compact support and helical symmetry.

In the next section we shall see that there are local solutions near null infinity which allow a Bondi expansion, but global source-free solutions have never a Bondi expansion.

\section{Global solutions with sources}

Suppose we have a solution of the form (9) defined near the center. In general, the series will not converge for all $r$. Examples are given by solutions of the inhomogeneous wave equation with sources invariant under the helical symmetry. The simplest case is a point source moving on an integral curve of the helical Killing field. In spacetime the retarded and advanced fields of this source are well defined outside the source. Near the center these solutions must be of the form (9) but they become singular at the source position. Further out we will again have a superposition of now both Bessel function solutions.

The general solution of the inhomogeneous wave equation with a spatially compact source is given by the sum of the retarded solution and some global solution of the homogeneous equation.
If the spatially compact source has helical symmetry the (unique) retarded and advanced solutions have also helical symmetry.

A simple way to see this is to consider a point charge as a source, as we do in detail in Section IV, and change the "source variables" $t^{\prime}, \phi^{\prime}$ to $t^{\prime}+\lambda$ and $\phi^{\prime}+\Omega \lambda, \lambda \in R$, which represents the action of the helical symmetry on the source. It is then easy to show, using the explicit formulae for Liénard-Wiechert potentials, that the field remains the same when the "observation variables" $t, \phi$ change to $t+\lambda, \phi+\Omega \lambda$. For an extended source the superposition principle can be used. There is, in fact, a more general theorem available showing for the wave equation that a symmetry of the source is inherited by the retarded/advanced fields.

If we take these helical solutions, which we obtain from general theorems about the wave equation in Minkowski space, and consider them as solutions of the reduced equation (2), we obtain immediately an existence theorem for the reduced equation which, in contrast to the result by Torre [10], includes the center.

More precisely: The retarded and advanced solution of the wave equation with a spatially compact helical source defines a global solution of the inhomogeneous reduced equation which is analytic outside the source. The general solution is obtained by adding a global solution of the homogeneous equation described in Section II B.

If we take a source with helical symmetry, then the difference of the retarded and advanced solution defines a global, source-free solution with helical symmetry on Minkowski space. This shows the existence of source-free solutions defined on all of Minkowski space.

We want to give an explicit representation of a helical solution with $Y_{l m}$-behaviour: Let $\rho$ be a helically symmetric source of the scalar field $\Phi(t, \mathbf{r})$, satisfying the inhomogeneous wave equation

$$
\square \Phi=-4 \pi \rho(t, \mathbf{r}),
$$

where $\mathbf{r}=\left(x^{i}\right)=(x, y, z)$. The retarded solution reads

$$
\Phi(t, \mathbf{r})=\int \frac{\rho(\bar{t}, \overline{\mathbf{r}})}{|\mathbf{r}-\overline{\mathbf{r}}|} d \bar{V}
$$

with

$$
\begin{gathered}
\bar{t}=t-|\mathbf{r}-\overline{\mathbf{r}}| \\
|\mathbf{r}-\overline{\mathbf{r}}|=\sqrt{r^{2}-2 \mathbf{r} \overline{\mathbf{r}}+\bar{r}^{2}}
\end{gathered}
$$

$r=|\mathbf{r}|=\left(x^{i} x^{i}\right)^{\frac{1}{2}}$, similarly for $\overline{\mathbf{r}}$.

We assume for the helical source the $Y_{l m}$ dependence:

$$
\begin{aligned}
\hat{\rho}(\hat{\phi}, r, \theta) & =\rho(r) Y_{l m}(\hat{\phi}, \theta)= \\
& =\rho(r) a_{l m} e^{i m \hat{\phi}} P_{l}^{m}(\cos \theta), \quad \hat{\phi} \in[0,2 \pi) .
\end{aligned}
$$

The integrand $I$ of the retarded integral becomes

$$
I=\frac{1}{|\mathbf{r}-\overline{\mathbf{r}}|} \rho(\bar{r}) a_{l m} e^{i m[\bar{\phi}-\Omega(t-|\mathbf{r}-\overline{\mathbf{r}}|)]} P_{l}^{m}(\cos \bar{\theta}) .
$$


So,

$$
I=\frac{\rho(\bar{r})}{|\mathbf{r}-\overline{\mathbf{r}}|} a_{l m} e^{i m \bar{\phi}} e^{-i m \Omega t} e^{i m \Omega|\mathbf{r}-\overline{\mathbf{r}}|} P_{l}^{m}(\cos \bar{\theta}),
$$

or

$$
I=e^{-i m \Omega t} \rho(\bar{r}) Y_{l m}(\bar{\phi}, \bar{\theta}) \frac{e^{i m \Omega|\mathbf{r}-\overline{\mathbf{r}}|}}{|\mathbf{r}-\overline{\mathbf{r}}|} .
$$

Now we can use the following expansion in spherical harmonics (see [14], Eqs.(16.18)-(16.22) with $m \Omega=k$ ):

$$
\begin{gathered}
\frac{e^{i k|\mathbf{r}-\overline{\mathbf{r}}|}}{4 \pi|\mathbf{r}-\overline{\mathbf{r}}|}=\sum_{l, m} g_{l}(r, \bar{r}) Y_{l m}^{*}(\bar{\theta}, \bar{\phi}) Y_{l m}(\theta, \phi), \\
g(r, \bar{r})=i k j_{l}\left(k r_{<}\right) h_{l}{ }^{(1)}\left(k r_{>}\right)
\end{gathered}
$$

with $r_{<}=\min (r, \bar{r}), r_{>}=\max (r, \bar{r})$. The Bessel and Hankel functions $j_{l}, h_{l}{ }^{(1)}$ can be found in 14] [Eqs.(16.9), (16.10)] or [15.

If we insert this expansion into the retarded integral, use the orthogonality properties of the spherical harmonics and remember the volume element we obtain

$$
\begin{aligned}
\Phi= & e^{-i m \Omega t} Y_{l m}(\phi, \theta) \\
& \times \int_{0}^{\infty} d \bar{r} \bar{r}^{2} \rho(\bar{r}) i m \Omega j_{l}\left(m \Omega r_{<}\right) h_{l}{ }^{(1)}\left(m \Omega r_{>}\right),
\end{aligned}
$$

or

$$
\begin{gathered}
\Phi=e^{-i m \Omega t} Y_{l m}(\phi, \theta) i m \Omega h_{l}{ }^{(1)}(m \Omega r) \int_{0}^{r} d \bar{r} \bar{r}^{2} \rho(\bar{r}) j_{l}(m \Omega \bar{r}) \\
+e^{-i m \Omega t} Y_{l m}(\phi, \theta) i m \Omega j_{l}(m \Omega r) \int_{r}^{\infty} d \bar{r} \bar{r}^{2} \rho(\bar{r}) h_{l}{ }^{(1)}(m \Omega \bar{r}) .
\end{gathered}
$$

For compactly supported $\rho(r)$ the behaviour near infinity is given by $h_{l}^{(1)}(m \Omega r) \sim e^{i m \Omega r} / m \Omega r$. In this representation of the solution the "retardation in space time" is completely hidden.

Thus, we have complete control of all solutions of the inhomogeneous reduced equation with signature change on the quotient. The lesson is not to forget Minkowski space and just work with the reduced equation on the quotient of the symmetry, but use all the results one has on the wave equation in Minkowski space!

\section{ASYMPTOTICS OF SOLUTIONS WITH SOURCES}

In this section we discuss the asymptotics of helical solutions on Minkowski space for scalar fields, and we also mention the analogous cases for Maxwell fields and linearized gravity. Here we deal with general (unspecified) sources of spatially compact support. In the next sections we investigate the fields of point sources on circular orbits in detail.

\section{A. The scalar wave equation}

Let $\rho$ be a helically symmetric source of the scalar field $\Phi(t, \mathbf{r})$, satisfying the inhomogeneous wave equation

$$
\square \Phi=-4 \pi \rho(t, \mathbf{r}),
$$

where $\mathbf{r}=\left(x^{i}\right)=(x, y, z)$. Again, the retarded solution reads (11). Assume now general $\rho$ which is bounded in space; then at large distance from the matter, $r \gg \bar{r}$, we obtain expansions for $\rho$ in (11).

We write

$$
|\mathbf{r}-\overline{\mathbf{r}}|=r+a(\bar{r}, \phi, \theta, \bar{\phi}, \bar{\theta})+O\left(\frac{1}{r}\right),
$$

and

$$
\frac{1}{|\mathbf{r}-\overline{\mathbf{r}}|}=\frac{1}{r}+O\left(\frac{1}{r^{2}}\right) .
$$

Because $\rho$ is helical we have

$$
\rho(\bar{t}, \bar{\phi}, \bar{\theta}, \bar{r})=\hat{\rho}(\bar{\phi}-\Omega \bar{t}, \bar{\theta}, \bar{r}),
$$

where $\hat{\rho}$ is periodic with period $2 \pi$ in its first argument. Hence we obtain

$$
\Phi(t, \phi, \theta, r)=\int \frac{\hat{\rho}(\bar{\phi}-\Omega(t-|\mathbf{r}-\overline{\mathbf{r}}|), \bar{\theta}, \bar{r})}{|\mathbf{r}-\overline{\mathbf{r}}|} d \bar{V} .
$$

Let us first investigate the field for $r \rightarrow \infty, t$ fixed. We can expand, using (23),

$$
\begin{gathered}
\hat{\rho}(\bar{\phi}-\Omega(t-|\mathbf{r}-\overline{\mathbf{r}}|), \bar{\theta}, \bar{r})= \\
=\hat{\rho}(\bar{\phi}-\Omega(t-r-a(\bar{r}, \phi, \theta, \bar{\phi}, \bar{\theta})), \bar{\theta}, \bar{r}) \\
+\frac{\partial \hat{\rho}}{\partial \bar{\phi}}(\bar{\phi}-\Omega(t-r+a(\bar{r}, \phi, \theta, \bar{\phi}, \bar{\theta})), \bar{\theta}, \bar{r}) \times O\left(\frac{1}{r}\right)+\ldots
\end{gathered}
$$

(We can easily write a complete Taylor expansion if needed.)

The leading contribution to the field is therefore [see also (24)]

$$
\begin{aligned}
& \Phi(t, \phi, \theta, r)= \\
= & \frac{1}{r} \int \hat{\rho}(\bar{\phi}-\Omega(t-r-a(\bar{r}, \phi, \theta, \bar{\phi}, \bar{\theta})), \bar{\theta}, \bar{r}) d \bar{V}+O\left(\frac{1}{r^{2}}\right) .
\end{aligned}
$$

The function

$$
P(t-r, \phi, \theta)=\int \hat{\rho}(\bar{\phi}-\Omega(t-r-a(\bar{r}, \phi, \theta, \bar{\phi}, \bar{\theta})), \bar{\theta}, \bar{r}) d \bar{V}
$$

is periodic in $t-r$ with period $2 \pi / \Omega$ and the asymptotic behaviour is oscillatory in $r$ for $t$ fixed, $r \rightarrow \infty$ :

$$
\Phi(t, \phi, \theta, r) \sim \frac{P(t-r, \phi, \theta)}{r} .
$$


For a helical source with $Y_{l m}$ behaviour,

$$
\hat{\rho}(\hat{\phi}, \theta, r)=e^{i m \hat{\phi}} P_{l}(\cos \theta) f(r),
$$

we can see the periodicity explicitly.

Next we consider the asymptotic behaviour at future null infinity $\mathcal{I}^{+}$. With $u=t-r$, we have

$$
\begin{aligned}
& \hat{\rho}(\bar{\phi}-\Omega(u+r-|\mathbf{r}-\overline{\mathbf{r}}|), \bar{\theta}, \bar{r})= \\
& \quad=\hat{\rho}(\bar{\phi}-\Omega(u-a(\bar{r}, \phi, \theta, \bar{\phi}, \bar{\theta})), \bar{\theta}, \bar{r})+ \\
& \quad+\frac{\partial \hat{\rho}}{\partial \bar{\phi}}(\bar{\phi}-\Omega(u-a(\bar{r}, \phi, \theta, \bar{\phi}, \bar{\theta})), \bar{\theta}, \bar{r}) \times O\left(\frac{1}{r}\right)+\ldots .
\end{aligned}
$$

If we put this in the retarded integral (11) we find that for $u=$ const, $r \rightarrow \infty$, we obtain the usual Bondi-type expansion in $r^{-1}$ (see [11], 12]) as we approach $\mathcal{I}^{+}$.

If we go to past null infinity $\mathcal{I}^{-}$, we have $v=t+r$ and obtain

$$
\begin{gathered}
\hat{\rho}(\bar{\phi}-\Omega(v+2 r-|\mathbf{r}-\overline{\mathbf{r}}|), \bar{\theta}, \bar{r})= \\
=\hat{\rho}(\bar{\phi}-\Omega(v+2 r-a(\bar{r}, \phi, \theta, \bar{\phi}, \bar{\theta})), \bar{\theta}, \bar{r})+ \\
+\frac{\partial \hat{\rho}}{\partial \bar{\phi}}(\bar{\phi}-\Omega(v+2 r-a(\bar{r}, \phi, \theta, \bar{\phi}, \bar{\theta})), \bar{\theta}, \bar{r}) \times O\left(\frac{1}{r}\right)+\ldots
\end{gathered}
$$

This for $r \rightarrow \infty, v=$ const implies again oscillations. This behaviour is easy to understand in a spacetime picture: when going to $\mathcal{I}^{-}$through the outgoing field of a source which has been periodically moving at all times, we cross infinitely many oscillations.

\section{B. The Maxwell field}

We take a continuous compact distribution of charges with helical motion. They define a conserved, helical 4current. If we use Lorentz gauge we have for each component of the 4-potential to solve a scalar wave equation with a helical source. Because the source is spatially compact the Lorentz condition is satisfied for the solutions given by the retarded, or advanced potentials. Hence, all the results for the scalar field apply.

\section{Linearized gravity}

If we take the $T^{\mu \nu}$ of particles moving along the orbits of a helical Killing vector we can consider the linearized equations in harmonic gauge. We then encounter again wave equations for individual components and can write down retarded and advanced solutions. The asymptotics is thus very similar to that of the scalar fields. However, now we cannot satisfy the harmonicity condition because the energy momentum tensor in linearized gravity for circular orbits is not conserved. A difference to the Maxwell case.

If we want a complete solution the matter must satisfy the equation of motion. For example, those of linearized elasticity.

\section{THE FIELD OF A POINT SCALAR CHARGE IN A CIRCULAR ORBIT}

We adopt the standard procedure of finding retarded and advanced Liénard-Wiechert potentials in electrodynamics (see, e.g., an elegant, covariant description by Rohrlich [16]) to the case of scalar fields. The retarded $(\epsilon=+1)$ and advanced $(\epsilon=-1)$ solutions of the wave equation with a $\delta$-function type source with scalar charge $Q$ moving along the worldline $\bar{x}^{\mu}(\tau)$ so that

$$
\rho\left(x^{\mu}\right)=Q \int \delta^{(4)}\left(x^{\mu}-\bar{x}^{\mu}(\tau)\right) d \tau,
$$

can be written in the form

$$
\Phi_{\epsilon}=-\frac{Q}{w_{\epsilon}},
$$

where

$$
w_{\epsilon}=-\epsilon \eta_{\alpha \beta} \frac{v_{\epsilon}^{\alpha}}{c} R_{\epsilon}^{\beta} .
$$

(Henceforth, we do not set set $c=1$.) Here the null vectors

$$
R_{\epsilon}^{\beta}=x^{\beta}-\bar{x}_{\epsilon}^{\beta}
$$

connect a given spacetime point $x^{\beta}$, in which $\Phi_{\epsilon}$ is to be calculated, with two points $\bar{x}_{\epsilon}^{\beta}$ on the worldline $\bar{x}^{\beta}(\tau)$, in which the past and future light cones with vertex at $x^{\beta}$ intersect the worldline. The $4-$ velocities $v_{\epsilon}^{\alpha}$ are evaluated at these points $\bar{x}_{\epsilon}^{\beta}$. The null vectors can be written as

$$
R_{\epsilon}^{\alpha}=\left(\epsilon R=\epsilon|\mathbf{R}|, \mathbf{R}=\mathbf{r}-\overline{\mathbf{r}}_{\epsilon}\right),
$$

the time components $R_{\epsilon}^{0}=\epsilon R$ are equal to the retarded and minus advanced distance of the particle to the field point $x^{\alpha}$.

Now consider a point charge $Q$ moving in the plane $z=0$ along the circular orbit of radius $a$ with center at $x=y=z=0$. So

$$
\overline{\mathbf{r}}=a\left(\cos \left(\Omega t+\phi_{0}\right), \sin \left(\Omega t+\phi_{0}\right), 0\right),
$$

where $\Omega=$ constant is the particle's angular velocity, $\phi_{0}$ is the azimuth at which the charge occurs at $t=0$. The $4-$ velocity is

$$
\begin{gathered}
v^{\mu}=(c \gamma, \gamma \mathbf{v}), \\
\gamma=\frac{1}{\sqrt{1-\frac{a^{2} \Omega^{2}}{c^{2}}}}=\text { constant }
\end{gathered}
$$

the 3 -velocity is

$$
\mathbf{v}=a \Omega\left(-\sin \left(\Omega t+\phi_{0}\right), \cos \left(\Omega t+\phi_{0}\right), 0\right) .
$$

From these simple relations we find the distance $R$ entering the null vectors (37) to be given by

$$
\begin{aligned}
R_{\epsilon}= & \left(\left(x-a \cos \left(\Omega t_{\epsilon}+\phi_{0}\right)\right)^{2}\right. \\
& \left.+\left(y-a \sin \left(\Omega t_{\epsilon}+\phi_{0}\right)\right)^{2}+z^{2}\right)^{\frac{1}{2}},
\end{aligned}
$$


which, in spherical coordinates, implies

$$
R_{\epsilon}=r\left(1-\frac{2 a}{r} \sin \theta \cos \left(\phi-\Omega t_{\epsilon}-\phi_{0}\right)+\frac{a^{2}}{r^{2}}\right)^{\frac{1}{2}} .
$$

Retarded and advanced times are implicitly given by the equation

$$
\begin{aligned}
t_{\epsilon} & =t-\epsilon \frac{R_{\epsilon}}{c} \\
& =t-\epsilon \frac{r}{c}\left(1-\frac{2 a}{r} \sin \theta \cos \left(\phi-\Omega t_{\epsilon}-\phi_{0}\right)+\frac{a^{2}}{r^{2}}\right)^{\frac{1}{2}}
\end{aligned}
$$

Note that on geometrical grounds we know that this equation has a unique solution for $t_{\epsilon}$ provided the worldline of the source is timelike, i.e., $\frac{a \Omega}{c}<1$. In contrast to the case of linear uniform or of uniformly accelerated motion, it is however impossible to obtain an explicit expression for $t_{\epsilon}$ as a function of $(t, r, \theta, \phi)$.

The expression in (36) for the scalar $w_{\epsilon}$ now reads

$$
\begin{gathered}
w_{\epsilon}=\gamma r\left(\left(1-\frac{2 a}{r} \sin \theta \cos \left(\phi-\Omega t_{\epsilon}-\phi_{0}\right)+\frac{a^{2}}{r^{2}}\right)^{\frac{1}{2}}-\right. \\
\left.-\epsilon \frac{a \Omega}{c} \sin \theta \sin \left(\phi-\Omega t_{\epsilon}-\phi_{0}\right)\right),
\end{gathered}
$$

which then implies directly the form of the resulting scalar field (35).

Let us finally rewrite the last relations in terms of the 'corotating' angular coordinate $\hat{\phi}=\phi-\Omega t$. Introducing

$$
\hat{\phi}_{\epsilon}=\phi-\Omega t_{\epsilon},
$$

the equation for the retarded/advanced time becomes

$$
\hat{\phi}_{\epsilon}=\phi+\epsilon \frac{\Omega r}{c}\left(1-\frac{2 a}{r} \sin \theta \cos \left(\hat{\phi}_{\epsilon}-\phi_{0}\right)+\frac{a^{2}}{r^{2}}\right)^{\frac{1}{2}} .
$$

Then the resulting scalar field,

$$
\begin{aligned}
\Phi_{\epsilon}=-\frac{Q}{\gamma r}\{ & \left(1-\frac{2 a}{r} \sin \theta \cos \left(\hat{\phi}_{\epsilon}-\phi_{0}\right)+\frac{a^{2}}{r^{2}}\right)^{\frac{1}{2}}- \\
& \left.-\epsilon \frac{a \Omega}{c} \sin \theta \sin \left(\hat{\phi}_{\epsilon}-\phi_{0}\right)\right\}^{-1}
\end{aligned}
$$

is independent of the time $t$.

Let us finally investigate the asymptotic behaviour of this solution. Define the following dimensionless quantities

$$
\begin{aligned}
& \mathcal{C}_{\epsilon}=\sin \theta \cos \left(\phi-\Omega t_{\epsilon}-\phi_{0}\right), \\
& \mathcal{S}_{\epsilon}=\sin \theta \sin \left(\phi-\Omega t_{\epsilon}-\phi_{0}\right), \\
& \mathcal{R}_{\epsilon}=\left(1-2 \mathcal{C}_{\epsilon} \frac{a}{r}+\frac{a^{2}}{r^{2}}\right)^{1 / 2},
\end{aligned}
$$

$$
\alpha=\frac{a \Omega}{c} .
$$

For a subluminal source, $\alpha<1$; in the non-relativistic limit, $\alpha \ll 1$. At large $r \gg a$ we find

$$
\mathcal{R}_{\epsilon}=1-\mathcal{C}_{\epsilon} \frac{a}{r}+\frac{1}{2} \mathcal{S}_{\epsilon}^{2} \frac{a^{2}}{r^{2}}+O\left(\left(\frac{a}{r}\right)^{3}\right),
$$

irrespective of $t, t_{\epsilon}, \theta, \phi$. This implies for the scalar field to be given at $r \gg a$ by the expansion

$$
\Phi_{\epsilon}=-\frac{Q}{\gamma r\left(1-\epsilon \alpha \mathcal{S}_{\epsilon}\right)}\left(1+\frac{\mathcal{C}_{\epsilon}}{\left(1-\epsilon \alpha \mathcal{S}_{\epsilon}\right)} \frac{a}{r}\right)+O\left(\left(\frac{a}{r}\right)^{3}\right) .
$$

For $\Omega=0$ we of course get the asymptotic static field of a source at rest at $r=a, \phi=\phi_{0}, \theta=\pi / 2$.

For a moving source we have to take into account equation (45) for the retarded/advanced time by employing the asymptotic form (54) for $\mathcal{R}_{\epsilon}$ :

$t_{\epsilon}=t-\epsilon \frac{r}{c} \mathcal{R}_{\epsilon}=t-\epsilon \frac{r}{c}+\epsilon \frac{a}{c} \mathcal{C}_{\epsilon}-\epsilon \frac{1}{2 c} \mathcal{S}_{\epsilon}^{2} \frac{a^{2}}{r^{2}}+O\left(\frac{1}{r^{2}}\right)$.

(If we drop the $O$-term we obtain a new, approximate implicit equation for $t_{\epsilon}$.) Fixing $t, \theta, \phi$, we get

$$
\left.\frac{\partial t_{\epsilon}}{\partial r}\right|_{t, \theta, \phi}=\frac{-\epsilon / c}{1-\epsilon \alpha \mathcal{S}_{\epsilon}}+O\left(\frac{1}{r}\right)
$$

Since $1-\epsilon \alpha \mathcal{S}_{\epsilon}>0, t_{\epsilon}$ is monotonically decreasing $(\epsilon=1)$ or increasing $(\epsilon=-1)$ function of $r$.

Consider first the limit $t$ fixed, $r \rightarrow \infty$ : The functions $\mathcal{C}_{\epsilon}, \mathcal{S}_{\epsilon}$ are oscillatory as $r \rightarrow \infty$ and, correspondingly, the scalar field decays, while oscillating, in accordance with Eq. (55). Retarded and advanced fields have similar asymptotic behaviour at spatial infinity $i_{0}$.

To see the character of the oscillations in more detail, we solve the equation for the dimensionless quantity $\Omega t_{\epsilon}$ iteratively (realizing that the term $\left|\epsilon \alpha \mathcal{C}_{\epsilon}\right|<1$ ). So,

$$
\Omega t_{\epsilon} \simeq \Omega t-\epsilon \frac{\Omega r}{c}+\epsilon \alpha \sin \theta \cos \left(\phi-\phi_{0}-\Omega\left(t-\epsilon \frac{r}{c}\right)\right) .
$$

The functions appearing in the fall-off of $\Phi_{\epsilon}$, with fixed $t, \theta, \phi$ and $r \rightarrow \infty$, thus behave as

$$
\mathcal{S}_{\epsilon}=p_{1} \sin \left(\epsilon \frac{\Omega}{c} r+\epsilon p_{2} \cos \left(\epsilon \frac{\Omega}{c} r+p_{3}\right)+p_{4}\right),
$$

$p_{1} \ldots p_{4}$ are constant parameters depending on fixed $t, \theta, \phi$; similarly for $\mathcal{C}_{\epsilon}$. Notice that $\mathcal{S}_{\epsilon}$ is again periodic in $r$ with the period $2 \pi c / \Omega$.

At null infinity, retarded and advanced fields behave very differently. Turning to future null infinity $\mathcal{I}^{+}$, we put $u=t-\frac{r}{c}$ and consider the limit $r \rightarrow \infty$, with $u, \theta, \phi$ fixed. From the asymptotic form of the equation (56) for $t_{\epsilon}$ for $r \rightarrow \infty, u, \theta, \phi$ fixed we now find that $\frac{\partial t_{+}}{\partial r}=O\left(r^{-1}\right)$, whereas $\frac{\partial t_{-}}{\partial r}=\frac{2}{c}\left(1-\alpha \mathcal{S}_{-}\right)^{-1}$. Here \pm is the abbreviation 
for $\epsilon= \pm$, i.e., retarded/advanced. Hence, $t_{+}$is approximately constant at large $r$, but $t_{-}$is a monotonically increasing function of $r$. Indeed, using the retarded time $t_{+}$from (58), we get $\mathcal{C}_{+}, \mathcal{S}_{+}$as functions of $u, \theta, \phi$, so that the retarded field given by (55) with $\epsilon=1$ has at $\mathcal{I}^{+}$the standard Bondi-type expansion [11], [12]

$$
\Phi_{+}=-\frac{Q}{\gamma}\left[\frac{f_{1}(u, \theta, \phi)}{r}+\frac{f_{2}(u, \theta, \phi)}{r^{2}}\right]+O\left(\frac{1}{r^{3}}\right) .
$$

However, the advanced field- 5 with $\epsilon=-1-$ contains functions like

$$
\mathcal{S}_{-}=k_{1} \sin \left(-\frac{2}{c} \Omega r+k_{2} \cos \left(-\frac{2}{c} \Omega r+k_{3}\right)+k_{4}\right),
$$

which oscillate in $r$ with period $\pi c / \Omega$, so half of that at spatial infinity.

At past null infinity $\mathcal{I}^{-}, r \rightarrow \infty$ with $v=t+\frac{r}{c}, \theta, \phi$ fixed, the advanced fields exhibit the standard Bonditype expansion whereas retarded fields decay oscillating in $r$. (All this becomes very clear in a spacetime picture.)

The oscillating factors at $r^{-k}$ in the asymptotic form (55) of $\Phi_{\epsilon}$ can become large for ultrarelativistic velocities when the source moves near the light cylinder.

For non-relativistic velocities, the asymptotics of the fields simplifies considerably. Assuming $\alpha \ll 1$ and neglecting terms which are $O\left(\alpha^{2}\right)$, we find the asymptotic expansion (55) to yield

$$
\begin{aligned}
& \Phi_{\epsilon}=- \frac{Q}{r}\left\{1+\epsilon \alpha \sin \theta \sin \left(\phi-\phi_{0}-\Omega\left(t-\epsilon \frac{r}{c}\right)\right)\right\} \\
&-\frac{Q a}{r^{2}}\left\{1+\epsilon 2 \alpha \sin \theta \sin \left(\phi-\phi_{0}-\Omega\left(t-\epsilon \frac{r}{c}\right)\right)\right\} \\
& \times\left\{\sin \theta \cos \left(\phi-\phi_{0}-\Omega\left(t-\epsilon \frac{r}{c}\right)\right)\right\}
\end{aligned}
$$

From this simple explicit expression the asymptotic behaviour of $\Phi_{\epsilon}$ at $i_{0}, \mathcal{I}^{ \pm}$becomes transparent. In particular it is easy to see that at the leading order near $i_{0}$ both $\Phi_{\epsilon}$ oscillate as $r^{-1} \sin (\epsilon \Omega r / c)$, whereas at $\mathcal{I}^{+}$the advanced field behaves like $r^{-1} \sin (2 \Omega r / c)$ and, in the same way, the retarded fields fall off at $\mathcal{I}^{-}$.

\section{THE FIELD OF A ELECTRIC POINT CHARGE IN A CIRCULAR ORBIT}

As mentioned above, in the Lorentz gauge the results for the individual components of the 4-potential will be essentially the same as for the scalar field. It is, however, of interest to see the behaviour of the electromagnetic field tensor because it determines directly the force and, from the perspective of the asymptotics, the peelingoff properties of its null tetrad projections. Since these may find applications also in other context, we shall give the null-tetrad components in the whole spacetime for a point charge moving along a circular orbit with, in general, relativistic speed (and so emitting synchrotron radiation).
We start from a general, covariant expression for the retarded/advanced field tensor of an electric point charge $e$ moving with a 4 -velocity $v^{\alpha}$ and a 4 -acceleration $a^{\alpha}$ [16. It is convenient to define a unit spacelike vector $u_{\alpha}$ which is orthogonal to $v^{\alpha}, u^{\alpha} u_{\alpha}=1, u^{\alpha} v_{\alpha}=0$, such that the null vectors $R_{\epsilon}^{\alpha}=x^{\alpha}-\bar{x}_{\epsilon}^{\alpha}$ (see Eqs. (37), (38)) can be written as

$$
R_{\epsilon}^{\alpha}=w_{\epsilon}\left(u_{\epsilon}^{\alpha}+\epsilon \frac{v_{\epsilon}^{\alpha}}{c}\right)
$$

Conversely,

$$
w_{\epsilon}=\eta_{\alpha \beta} u_{\epsilon}^{\alpha} R_{\epsilon}^{\beta}=-\epsilon \eta_{\alpha \beta} \frac{v_{\epsilon}^{\alpha}}{c} R_{\epsilon}^{\beta},
$$

where the last expression is identical with (36). The $4-$ potential is simply

$$
A_{\epsilon}^{\alpha}=\frac{e}{c} \frac{v_{\epsilon}^{\alpha}}{w_{\epsilon}}
$$

which shows that it differs from the scalar field $\Phi_{\epsilon}$ given in (35) just by the replacement $Q \rightarrow-\frac{e}{c} v^{\alpha}$. The resulting expression for the Maxwell field tensor reads:

$$
\begin{aligned}
F_{\epsilon}^{\mu \nu}= & \frac{e}{c w_{\epsilon}^{2}} v_{\epsilon}^{[\mu} u_{\epsilon}^{\nu]} \\
& +\frac{e}{c^{2} w_{\epsilon}}\left(\frac{1}{c} a_{\epsilon}^{[\mu} v_{\epsilon}^{\nu]}-u_{\epsilon}^{[\mu}\left(\frac{1}{c} v_{\epsilon}^{\nu]} a_{\epsilon}+\epsilon a_{\epsilon}^{\nu]}\right)\right)
\end{aligned}
$$

where the small square brackets denote antisymmetrization without " $\frac{1}{2} ", a_{\epsilon}:=\eta_{\alpha \beta} a_{\epsilon}^{\alpha} u_{\epsilon}^{\beta}$.

For the circular motion with the same parameters as with the scalar charge all expressions for the velocity, retarded and advanced times and the scalar $w_{\epsilon}$ are again given by formulas (39)- (46). In the expression (66) we also need the 4-acceleration $a^{\mu}=d v^{\mu} / d \tau=\gamma d v^{\mu} / d t$, where $v^{\mu}$ is given by (40)-(42); the result is

$$
a_{\epsilon}^{\mu}=-\frac{a \Omega^{2}}{1-\frac{a^{2} \Omega^{2}}{c^{2}}}\left(0, \cos \left(\Omega t_{\epsilon}+\phi_{0}\right), \sin \left(\Omega t_{\epsilon}+\phi_{0}\right), 0\right) \text {. }
$$

From 63 the spacelike vector $u_{\epsilon}^{\mu}$ can be expressed as $u_{\epsilon}^{\mu}=R_{\epsilon}^{\mu} w_{\epsilon}{ }^{-1}-\epsilon v_{\epsilon}^{\mu} / c$, which in case of the circular motion implies the components to be

$$
\begin{aligned}
u_{\epsilon}^{0}= & \epsilon w_{\epsilon}^{-1} r R_{\epsilon}-\epsilon \gamma \\
u_{\epsilon}^{1}= & w_{\epsilon}^{-1}\left(r \sin \theta \cos \phi-a \cos \left(\Omega t_{\epsilon}+\phi_{0}\right)\right)+ \\
& \left.+\frac{\epsilon}{c} \gamma a \Omega \sin \left(\Omega t_{\epsilon}+\phi_{0}\right)\right) \\
u_{\epsilon}^{2}= & w_{\epsilon}^{-1}\left(r \sin \theta \sin \phi-a \sin \left(\Omega t_{\epsilon}+\phi_{0}\right)\right)- \\
& \left.-\frac{\epsilon}{c} \gamma a \Omega \cos \left(\Omega t_{\epsilon}+\phi_{0}\right)\right) \\
u_{\epsilon}^{3}= & w_{\epsilon}^{-1} r \cos \theta
\end{aligned}
$$

it is easy to check that $u_{\epsilon}^{\mu}$ is unit and perpendicular to $v_{\epsilon}^{\mu}$. The last necessary ingredient is the projection $a_{\epsilon}$ of $a_{\epsilon}^{\mu}$ on $u_{\epsilon}^{\mu}$ :

$$
a_{\epsilon}=-w_{\epsilon}^{-1} a^{2} \Omega^{2} \gamma^{2}\left(\frac{r}{a} \sin \theta \cos \left(\phi-\Omega t_{\epsilon}-\phi_{0}\right)-1\right) .
$$


The 4-potential is determined by (65) in terms of the quantities we know already from the scalar field case the scalar $w_{\epsilon}$, see (46), and by the 4 -velocity $v_{\epsilon}^{\mu}$ given by (40)-(42) with $t \rightarrow t_{\epsilon}$. Notice that the time-component $A_{\epsilon}^{t}$ is equal to the scalar field $\Phi_{\epsilon}$ [see [35)] up to the sign and the multiplicative $\gamma$ factor.

For highly relativistic motions, $A_{\epsilon}^{t}$ is thus much bigger than the corresponding $\Phi_{\epsilon}$. The asymptotics of $A_{\epsilon}^{t}$ is, however, the same as of $\Phi_{\epsilon}$ (see (55), (60) and (62)). Owing to the symmetry, $A_{\epsilon}^{z}=0$. Because of the velocity entering the componets $A_{\epsilon}^{x}, A_{\epsilon}^{y}$, their asymptotic expansion at $i_{0}$ begins immediately with the oscillatory terms at $r^{-1}$ even for non-relativistic motions $(\alpha \ll 1)$, rather than with constant $\times r^{-1}$ as in (62), and the same with the advanced (retarded) fields at $\mathcal{I}^{+}\left(\mathcal{I}^{-}\right)$.

Substituting for $w_{\epsilon}, v_{\epsilon}^{\mu}, a_{\epsilon}^{\mu}, u_{\epsilon}^{\mu}$ into (66) we obtain all components of the Maxwell field tensor. These are quite lengthy, here we give just the null tetrad components from which all information can be retrieved. The standard null (Newman-Penrose) tetrad (see, e.g., [17]) in Minkowski spacetime reads [18]

$$
\begin{aligned}
& l^{\mu}=\frac{1}{\sqrt{2}}(1, \cos \phi \sin \theta, \sin \phi \sin \theta, \cos \theta) \\
& n^{\mu}= \frac{1}{\sqrt{2}}(1,-\cos \phi \sin \theta,-\sin \phi \sin \theta,-\cos \theta), \\
& m^{\mu}= \frac{1}{\sqrt{2}}(0, \cos \phi \cos \theta-i \sin \phi \\
&\quad \sin \phi \cos \theta+i \cos \phi,-\sin \theta)
\end{aligned}
$$

in our signature it satisfies $l^{\mu} n_{\mu}=-1, m^{\mu} \bar{m}_{\mu}=1$, all vectors are null.

Now the null tetrad electromagnetic scalars are given as the following projections of the Maxwell tensor:

$$
\begin{aligned}
\Phi_{0} & =F_{\mu \nu} l^{\mu} m^{\nu}, \\
\Phi_{1} & =\frac{1}{2} F_{\mu \nu}\left(l^{\mu} n^{\nu}+\bar{m}^{\mu} m^{\nu}\right), \\
\Phi_{2} & =F_{\mu \nu} \bar{m}^{\mu} n^{\nu} .
\end{aligned}
$$

The null tetrad naturally induces the orthonormal tetrad (see Ref. [18]) associated with the spherical coordinates with the standard orthonormal triad $e_{\hat{r}}, e_{\hat{\theta}}, e_{\hat{\phi}}$ (supplemented by the timelike vector $(1,0,0,0))$. From the scalars $\Phi_{q}, q=0,1,2$ the physical components of the electric and magnetic field in the triad can be expressed by means of the relation

$$
\begin{aligned}
E_{\hat{r}}-i B_{\hat{r}} & =2 \phi_{1} \\
E_{\hat{\theta}}-i B_{\hat{\theta}} & =-\Phi_{0}+\Phi_{2} \\
E_{\hat{\phi}}-i B_{\hat{\phi}} & =-i\left(\Phi_{0}+\Phi_{2}\right)
\end{aligned}
$$

The Maxwell tensor field is given by (66), with all the ingredients expressed explicitly in (39)-(46), (67)-(72). In addition to $\mathcal{C}_{\epsilon}, \mathcal{S}_{\epsilon}, \mathcal{R}_{\epsilon}$ and $\alpha$ determined by (50)-(53), we introduce another dimensionless quantity

$$
\mathcal{Q}_{\epsilon}=\mathcal{R}_{\epsilon}-\epsilon \alpha \mathcal{S}_{\epsilon}=\left(1-2 \mathcal{C}_{\epsilon} \frac{a}{r}+\frac{a^{2}}{r^{2}}\right)^{\frac{1}{2}}-\epsilon \alpha \mathcal{S}_{\epsilon} .
$$

Finally, substituting the explicit expression for $F_{\epsilon}^{\mu \nu}$ and the null tetrad (73) into (74), we obtain -after straightforward though not very short calculations and arrangements - the following results for the 3 complex scalars $\Phi_{0}, \Phi_{1}, \Phi_{2}$, in which again $\hat{\phi}_{\epsilon}=\phi-\Omega t_{\epsilon}$ :

$$
\begin{aligned}
\Phi_{0}= & \frac{e \Omega}{2 c r \mathcal{Q}_{\epsilon}^{3}}\left\{-\alpha\left(1-\epsilon \mathcal{R}_{\epsilon}-\mathcal{C}_{\epsilon} \frac{a}{r}+\frac{a^{2}}{r^{2}}\right)\left[\cos \theta \cos \left(\hat{\phi}_{\epsilon}-\phi_{0}\right)-i \sin \left(\hat{\phi}_{\epsilon}-\phi_{0}\right)\right]\right. \\
& \left.+(1-\epsilon \mathcal{R}) \frac{a}{r}\left[-\cos \theta \sin \left(\hat{\phi}_{\epsilon}-\phi_{0}\right)-i \cos \left(\hat{\phi}_{\epsilon}-\phi_{0}\right)\right]+i\left[\alpha^{2}\left(1-\epsilon \mathcal{R}_{\epsilon}-\mathcal{C}_{\epsilon} \frac{a}{r}\right)+\frac{a^{2}}{r^{2}}\right] \sin \theta\right\}+ \\
& +\frac{e a}{2\left(r \mathcal{Q}_{\epsilon}\right)^{3}}\left[\cos \theta \cos \left(\hat{\phi}_{\epsilon}-\phi_{0}\right)-i \sin \left(\hat{\phi}_{\epsilon}-\phi_{0}\right)\right], \\
\Phi_{1}= & \frac{e}{2 r^{2} \mathcal{Q}_{\epsilon}^{3}}\left\{1-\epsilon \alpha \mathcal{R}_{\epsilon} \sin \theta \sin \left(\hat{\phi}_{\epsilon}-\phi_{0}\right)-\left(\alpha^{2} \mathcal{C}_{\epsilon}+\left(1-\alpha^{2}\right) \frac{a}{r}+i \alpha^{3} \cos \theta\right) \sin \theta \cos \left(\hat{\phi}_{\epsilon}-\phi_{0}\right)+i \alpha \frac{a}{r} \cos \theta\right\}, \\
\Phi_{2}= & -\frac{e \Omega}{2 c r \mathcal{Q}_{\epsilon}^{3}}\left\{-\alpha\left(1+\epsilon \mathcal{R}_{\epsilon}-\mathcal{C}_{\epsilon} \frac{a}{r}+\frac{a^{2}}{r^{2}}\right)\left[\cos \theta \cos \left(\hat{\phi}_{\epsilon}-\phi_{0}\right)+i \sin \left(\hat{\phi}_{\epsilon}-\phi_{0}\right)\right]\right. \\
& \left.+\left(1+\epsilon \mathcal{R}_{\epsilon}\right) \frac{a}{r}\left[\cos \theta \sin \left(\hat{\phi}_{\epsilon}-\phi_{0}\right)-i \cos \left(\hat{\phi}_{\epsilon}-\phi_{0}\right)\right]+i \sin \theta\left[\alpha^{2}\left(1+\epsilon \mathcal{R}_{\epsilon}-\mathcal{C}_{\epsilon} \frac{a}{r}\right)+\frac{a^{2}}{r^{2}}\right]\right\} \\
& -\frac{e a}{2\left(r \mathcal{Q}_{\epsilon}\right)^{3}}\left[\cos \theta \cos \left(\hat{\phi}_{\epsilon}-\phi_{0}\right)-i \sin \left(\hat{\phi}_{\epsilon}-\phi_{0}\right)\right] .
\end{aligned}
$$


There are well-known properties of the null-tetrad components of the zero-rest mass fields (see, e.g., [12]). In the electromagnetic case $\Phi_{2}$ characterizes the outgoing radiation field at $\mathcal{I}^{+}$: it is given as $\lim _{r \rightarrow \infty, u=c o n s t}\left(r \Phi_{2}\right)$. At $\mathcal{I}^{-} \lim _{r \rightarrow \infty, v=\text { const }}\left(r \Phi_{0}\right)$ determines the incoming radiation field. We shall see that, indeed, this is the case for $\Phi_{2+}$ at $\mathcal{I}^{+}$and for $\Phi_{0-}$ at $\mathcal{I}^{-}$. (Here \pm is again the abbreviation for $\epsilon= \pm 1$, i.e., retarded/advanced fields.)

Before turning to the asymptotic properties of the $\Phi_{\mathrm{s}}$ let us observe some intriguing "symmetry relations" of these quantities. They arise as a consequence of the helical symmetry of the source and the fields. In order to understand them we have to turn back to the equation (45) for retarded and advanced times. Notice first that putting the 'field' time $t=0$, Eq. (45) still cannot be solved explicitly for $t_{\epsilon}$, however it is easy to see the following relations between $t_{+}$and $t_{-}$:

$$
t_{+}(t=0, r, \theta, \phi)=-t_{-}\left(t=0, r, \theta,-\phi+2 \phi_{0}\right) .
$$

(It is instructive to draw the circular orbit of the source and convince oneself that this relation can also be understood on "geometrical grounds".) Owing to the helical symmetry the relation (811) can be generalized for any $t$ to read

$$
t_{+}(t, r, \theta, \phi+\Omega t)-t=-t_{-}\left(t, r, \theta,-\phi+\Omega t+2 \phi_{0}\right)+t ;
$$

this can be checked directly using (45) again.

Now as a consequence of helical symmetry and, in particular, of (82), there arise the following relations - valid at any spacetime point - between the retarded and advanced fields as given by the null-tetrad components of the $\Phi$ 's:

$$
\Phi_{2+}(t, r, \theta, \phi+\Omega t)=-\Phi_{0-}\left(t, r, \theta,-\phi+\Omega t+2 \phi_{0}\right),
$$

$$
\begin{aligned}
& \Phi_{0+}(t, r, \theta, \phi+\Omega t)=-\Phi_{2-}\left(t, r, \theta,-\phi+\Omega t+2 \phi_{0}\right), \\
& \Phi_{1+}(t, r, \theta, \phi+\Omega t)=+\Phi_{1-}\left(t, r, \theta,-\phi+\Omega t+2 \phi_{0}\right) .
\end{aligned}
$$

It is straightforward to convince oneself that our resulting expressions (77)-(79) satisfy these relations. They are the keystone in Schild's 2-body problem, in fact the $N$-body as well, implying that tangential forces on particles vanish when both retarded and advanced effects are taken into account. But this problem will be considered elsewhere (for the $N$-particle problem in the case of the scalar field, see [4]).

We now turn to the asymptotic properties of the fields. As with the scalar field we first make the expansions of the retarded and advanced fields at large $r \gg a$ using the expansion (54) of $\mathcal{R}_{\epsilon}$. In addition to the the abbreviations $\mathcal{C}_{\epsilon}, \mathcal{S}_{\epsilon}$ defined in (50), (51), i.e.,

we introduce

$$
\begin{aligned}
\mathcal{C}_{\epsilon} & =\sin \theta \cos \left(\hat{\phi}_{\epsilon}-\phi_{0}\right), \\
\mathcal{S}_{\epsilon} & =\sin \theta \sin \left(\hat{\phi}_{\epsilon}-\phi_{0}\right),
\end{aligned}
$$

$$
\begin{aligned}
& \tilde{\mathcal{C}}_{\epsilon}=\cos \theta \cos \left(\hat{\phi}_{\epsilon}-\phi_{0}\right), \\
& \tilde{\mathcal{S}}_{\epsilon}=\cos \theta \sin \left(\hat{\phi}_{\epsilon}-\phi_{0}\right),
\end{aligned}
$$

and

$$
\begin{aligned}
& c_{\epsilon}=\cos \left(\hat{\phi}_{\epsilon}-\phi_{0}\right), \\
& s_{\epsilon}=\sin \left(\hat{\phi}_{\epsilon}-\phi_{0}\right) .
\end{aligned}
$$

With these notations we obtain the following:

$$
\begin{aligned}
\Phi_{2+}= & \frac{e \Omega \alpha}{c\left(1-\alpha \mathcal{S}_{+}\right)^{3}}\left(\tilde{\mathcal{C}}_{+}+i s_{+}-i \alpha \sin \theta\right) \frac{1}{r}+O\left(\frac{1}{r^{2}}\right) \\
\Phi_{2-}= & -\frac{e a}{2\left(1+\alpha \mathcal{S}_{-}\right)^{3}}\left\{\tilde{\mathcal{C}}_{-}+i s_{-}+\alpha\left[C_{-}\left(\tilde{\mathcal{S}}_{-}-i c_{-}\right)+\right.\right. \\
& \left.\left.+i \sin \theta-\frac{1}{2} \alpha\left(1+\mathcal{C}_{-}^{2}\right)\left(\tilde{\mathcal{C}}_{-}+i s_{-}\right)-i \frac{\alpha^{2}}{2}\left(1-\mathcal{C}_{-}^{2}\right) \sin \theta\right]\right\} \frac{1}{r^{3}}+O\left(\frac{1}{r^{4}}\right), \\
\Phi_{1 \epsilon}= & \frac{e}{2\left(1-\epsilon \alpha \mathcal{S}_{\epsilon}\right)^{3}}\left[1-\epsilon \alpha \mathcal{S}_{\epsilon}-\left(\alpha^{2} \mathcal{C}_{\epsilon}+i \alpha^{2} \cos \theta\right) \mathcal{C}_{\epsilon}\right] \frac{1}{r^{2}}+O\left(\frac{1}{r^{3}}\right) \\
\Phi_{0+}= & -\frac{e a}{2\left(1-\alpha \mathcal{S}_{+}\right)^{3}}\left\{\tilde{\mathcal{C}}_{+}-i s_{+}+\alpha\left[-\mathcal{C}_{+}\left(\tilde{\mathcal{S}}_{+}-i c_{+}\right)+\right.\right. \\
& \left.\left.+i \sin \theta-\frac{1}{2} \alpha\left(1+\mathcal{C}_{+}^{2}\right)\left(\tilde{\mathcal{C}}_{+}-i s_{+}\right)-i \frac{\alpha^{2}}{2}\left(1-\mathcal{C}_{+}^{2}\right) \sin \theta\right]\right\} \frac{1}{r^{3}}+O\left(\frac{1}{r^{4}}\right), \\
\Phi_{0-}= & -\frac{e \Omega \alpha}{c\left(1+\alpha \mathcal{S}_{-}\right)^{3}}\left(\tilde{\mathcal{C}}_{-}-i s_{-}-i \alpha \sin \theta\right) \frac{1}{r}+O\left(\frac{1}{r^{2}}\right) .
\end{aligned}
$$


These formulas get still simplified for nonrelativistic motion of the source, $\alpha \ll 1$, when only terms linear in $\alpha$ are kept (using $\left.\left(1-\epsilon \alpha \mathcal{S}_{\epsilon}\right)^{-3} \simeq 1+3 \alpha \mathcal{S}_{\epsilon}+O\left(\alpha^{2}\right)\right)$ and rearranging the terms:

$$
\begin{gathered}
\Phi_{2+}=\frac{e \Omega \alpha}{c}\left(\tilde{\mathcal{C}}_{+}+i s_{+}\right) \frac{1}{r}+O\left(\frac{1}{r^{2}}\right) \\
\Phi_{2-}=-\frac{e a}{2}\left[\left(1-2 \alpha \mathcal{S}_{-}\right)\left(\tilde{\mathcal{C}}_{-}+i s_{-}\right)\right] \frac{1}{r^{3}}+O\left(\frac{1}{r^{4}}\right) \\
\Phi_{1 \epsilon}=\frac{e}{2}\left(1+2 \epsilon \alpha \mathcal{S}_{\epsilon}\right) \frac{1}{r^{2}}+O\left(\frac{1}{r^{3}}\right) \\
\Phi_{0+}=\frac{e a}{2}\left[\left(1+2 \alpha \mathcal{S}_{+}\right)\left(\tilde{\mathcal{C}}_{+}-i s_{+}\right)\right] \frac{1}{r^{3}}+O\left(\frac{1}{r^{4}}\right) \\
\Phi_{0-}=-\frac{e \Omega \alpha}{c}\left(\tilde{\mathcal{C}}_{-}-i s_{-}\right) \frac{1}{r}+O\left(\frac{1}{r^{2}}\right)
\end{gathered}
$$

Clearly, the fields have again the oscillatory character. The retarded and advanced times $t_{\epsilon}$ can be expressed in terms of the field time $t$ and functions $\mathcal{C}_{\epsilon}$ and $\mathcal{S}_{\epsilon}$ according to Eq. (56). At fixed $t, \theta, \phi$ and $r \rightarrow \infty$, i.e., at $i_{0}$, the oscillations are given by $\mathcal{C}_{\epsilon}, \mathcal{S}_{\epsilon}$ which have the form (59) as in the scalar-field case. The retarded and advanced fields behave similarly.

At future null infinity $\mathcal{I}^{+}$the retarded field has the standard Bondi-type expansion since $\mathcal{C}_{+}, \mathcal{S}_{+}$become functions of $u, \theta, \phi$ [see Eq. (60) and the text therein]. In addition, in the electromagnetic case we observe a new feature in the asymptotics - the well-known "peeling-off property" [12], [17]: $\Phi_{q+} \sim f_{q}(u, \theta, \phi) r^{q-3}$. The limits $r^{3-q} \Phi_{q+}$ are well defined at $r \rightarrow \infty$ with $u=t-r / c, \theta, \phi$ fixed, i.e., at $\mathcal{I}^{+}$. With the advanced field at $\mathcal{I}^{+}$the situation is different. We still find a "generalized peeling" in the sense that $\Phi_{q-} \sim r^{-1-q}$; so $\Phi_{q-}$ corresponds to $\Phi_{(2-q)+}$. However, the limits $r^{1+q} \Phi_{q-}$ at $r \rightarrow \infty, u, \theta, \phi$ fixed do not exist since these are oscillatory functions as $\mathcal{S}_{-}$in (61), i.e., with period $\pi c / \Omega$ which is half of that at spatial infinity. Nevertheless, notice that $r^{1+q} \Phi_{q-}$ remains bounded as $r \rightarrow \infty$.

As expected, at past null infinity $\mathcal{I}^{-}, r \rightarrow \infty, v=t+$ $r / c, \theta, \phi$ fixed, the advanced fields exhibit the standard Bondi-type expansion and peeling, whereas the retarded fields do decay with $r \rightarrow \infty$ but in an oscillatory manner.

All these features become evident especially when regarding the simple fields (95)-(99) in the non-relativistic limit $\alpha \ll 1$.

\section{ACKNOWLEDGMENTS}

We thank J. Winicour for many useful discussions. J.B. gratefully acknowledges the support of the Alexander von Humboldt Foundation and also of the grants No. GACR 202/06/0041, No. LC06014 and MSM0021620860 of the Czech Rebublic.
[1] L. Lichtenstein, Mathematische Zeitschrift 12, 201 (1922).

[2] J. K. Blackburn and S. Detweiler, Phys. Rev. D 46, 2318 (1992). S. Detweiler, Phys. Rev. D 50, 4929 (1994).

[3] A. Schild, Phys.Rev. 131(6), 2762 (1963).

[4] R. Beig, J. M. Heinzle, and B. G. Schmidt, Phys. Rev. Lett., to be published (2007).

[5] J. L. Friedman, K. Uryū, and M. Shibata, Phys. Rev. D 65, 064035 (2002); 70, 129904(E) (2004);

J. L. Friedman and K. Uryū, Phys. Rev. D 73, 104039 (2006);

S. Yoshida, B. C. Bromley, J. S. Read, K. Uryu, and J. L. Friedman, Class. Quantum Grav. 23, S599 (2006).

[6] J. T. Whelan, W. Krivan, and R. H. Price, Class. Quantum Grav. 17, 4895 (2000);

B. Bromley, R. Owen, and R. H. Price, Phys. Rev. D 71, 104017 (2005);

C. Beetle, B. Bromley, and R. H. Price, Phys. Rev. D 74, 024013 (2006).

[7] E. Gourgoulhon, P. Grandclement, and S. Bonazzola, Phys. Rev. D 65, 044020 (2002);

T. Damour, E. Gourgoulhon, and P. Grandclement, Phys. Rev. D 66, 024007 (2002);
E. Gourgoulhon, P. Grandclement, and S. Bonazzola, Int. J. Mod. Phys. A17, 2689 (2002).

[8] C. Klein, Phys. Rev. D 70, 124026 (2004).

[9] J. P. Bruneton, gr-qc/0611021, to appear in the proceedings of Albert Einstein's Centunary International Conference, Paris, France, 18-22 July; J. P. Bruneton and G. Esposito-Farèse (unpublished).

[10] C. G. Torre, J. Math. Phys. 44, 6223 (2003); J. Math. Phys. 47, 073501 (2006).

[11] H. Bondi, M. G. J. van der Burg, and A. W. K. Metzner, Proc. Roy. Soc. Lond. A269, 21 (1962).

[12] R. Penrose, Proc. Roy. Soc. Lond. A284, 159 (1965).

[13] R. M. Wald, General Relativity (Univ. Chicago Press, Chicago, 1984).

[14] J. D. Jackson Classical electrodymics, 2nd Edition (John Wiley, New York 1975).

[15] M. Abramowitz and I. A. Stegun, Handbook of Mathematical Functions (Dover Publ., New York 1972).

[16] F. Rohrlich, Classical Charged Particles (AddisonWesley, Reading, 1996).

[17] J. Stewart, Advanced general relativity (Cambridge University Press, Cambridge, U.K., 1991).

[18] This null tetrad is adapted to the spherical coordi- 
nate lines rather than to Cartesian coordinates but its components are Minkowskian expressed in terms of $t, r, \theta, \phi$. The associated standard tetrad is given by $e_{(0)}^{\mu}=(1 / \sqrt{2})\left(l^{\mu}+n^{\mu}\right), e_{(\hat{r})}^{\mu}=(1 / \sqrt{2})\left(l^{\mu}-n^{\mu}\right), e_{(\hat{\theta})}^{\mu}=$
$(1 / \sqrt{2})\left(m^{\mu}+\bar{m}^{\mu}\right), e_{(\hat{\phi})}^{\mu}=(i / \sqrt{2})\left(m^{\mu}-\bar{m}^{\mu}\right)$. In spherical coordinates, $e_{(\hat{r})}=(0,1,0,0), e_{(\hat{\theta})}=(0,0,1 / r, 0), e_{(\hat{\phi})}=$ $(0,0,0,1 / r \sin \theta)$. 